\title{
A Brief Review of Breast Cancer Detection via Computer Aided Deep Learning Methods
}

\author{
Ayush Dogra \\ UIET, Panjab University \\ Chandigarh, India
}

\author{
Bhawna Goyal \\ UIET, Panjab University \\ Chandigarh, India
}

\author{
Kaushik K \\ Srinivas Institute of Technology \\ Mangluru, India
}

\begin{abstract}
Breast cancer has emerged as a leading cause of death in women around the globe. The detection of early onset of breast cancer is very important. The CAD (computer aided diagnosis) have surfaced as an assistive medical diagnostic tool which help a great deal in patient care and management by reducing the ratio of false positive results and by enabling a speedy diagnosis. The rapid progression in the development of highly sophisticated deep learning based image processing methods has facilitated the computer aided automatic detection of breast cancer. In this manuscript we have presented a brief review of the most recent trends in deep learning based breast cancer detection methods. This paper besides laying a general framework of a CAD tool also eludes upon various preprocessing and segmentation methods.
\end{abstract}

Keywords - Medical image, breast cancer, deep learning, machine learning, lesion, classifiers

\section{INTRODUCTION}

Breast cancer has become one of the leading causes of death in women in recent past. Cancer can be termed as an abnormal growth, where some cells tend to grow in an aberrant manner. Due to the anatomy of human body women are much more vulnerable to breast cancer. Breast cancer occurs when tissues of breast starts getting uncontrollably divided. In the year 2019, 42260 deaths were reported due to breast cancer (American Society of Cancer). According to [1], with an annual incidence rate of 144000 new cases of breast cancer, it has become the most common type of female cancer in urban India. It proliferates through lobules (tissues containing glands for milk production) to ducts which joins lobules to nipples. Breast also contains connective, lymphatic and fatty tissues.

Early detection and visualisation of cancer hence becomes utterly important for saving lives of people. Breast cancer is typically diagnosed through a screening examination before the symptoms have developed or when a woman suspects a lump. Most lumps visualized in radiographic imaging are benign in nature and do not grow in an uncontrollable manner. However if a lesion or tumour is suspected after imaging, microscopic analysis of breast tissue are essential. Mammography, Magnetic resonance imaging, sonography, histopathical images, computed tomography and physical examinations are most commonly employed diagnostic tools for breast cancer. Among them Full Field Digital Mammography, nuclear medicine, thermographs and contrast enhanced digital mammography are new emerging modalities for cancer care and management. For an assistive screening and detection, one anticipates continuous linear growth of tumour. However if such assumptions are not correct and tumour depicts a heterogenic growth, this can lead to false positive diagnosis [2-5]. The false diagnosis results in extreme patient discomfort, increased expenditure and further psychological implications. Also tumour is an extremely element of medical investigation. Even expert radiologists fail to sometimes interpret the exact scenario. Hence the CAD tool have become indispensable in order to assist the radio-diagnosis in the field of breast related oncology.

In current scenario, computer aided analysis is readily employed as pattern recognition software which helps in identifying the abnormalities (malignant or benign) and marks them for the radiologists. In a study given in [6 7,] CAD analysis helps in reducing the false negative rate by $77 \%$. It was also found that $\mathrm{CAD}$ systems are quite accurate in detecting microcalcifications, ductal carcinoma in situ and invasive lobular carcinoma. The most popular CAD system is the R2 Image Checker.

Due to the growing popularity of the CAD models and tools various investigation methods and algorithms have been designed by researchers worldwide in order to analyse breast images from different perspectives depending on the type of disease. Basically the concept of machine learning (ML) and artificial intelligence has largely influenced this field of biomedical engineering. A ML-CAD model is comprised of extraction of the desired feature followed by classification via learned machine. A general framework for breast image cancer detection can be summarised as follows $[3,8,9]$ :

I.Selecting and extracting the feature detail quantized by shape, intensity and texture of suspected region

\section{II.CLASSIFIER MODEL}

\section{III.Performance Evaluation parameter}

IV.Output via classifier.

An efficient machine learning CAD tool consists of preprocessing of source images, image segmentation and feature extraction and post processing. For a ML-CAD tool to work optimally feature extraction is the key step. Deep learning based methods gave an advantage of generating high level feature representation directly from raw images. In order to establish the user friendly framework of these CAD tools, Graphical Processing Unit (GPU) are being employed in parallel in order to facilitate recognition of images and extraction of features. For the design and development of these tools there are wide range of datasets which are publicly available which include BreakHis, INbreast, Danish Lung Cancer Screening Trail (DLCST), MITOSTAPIA and Image Database resource initiative (IDRI) [1-8]. The various cancer detection stages can be understood as: (1) Pre-processing of noisy raw source images, contrast enhancement, colour correction and hair removal. (2) Image 
segmentation using supervised learning, histogram thresholding and probabilistic modelling and graph theory. (3) Post processing employing morphological operations and smoothing.

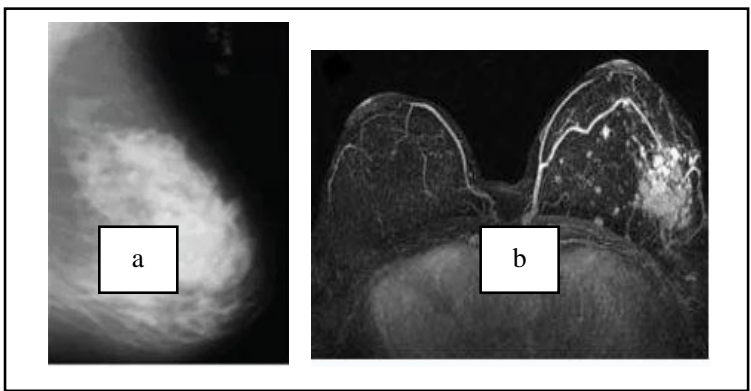

Fig 1 (a) shows a breast mammogram; (b) breast MRI [9]

Table 1: DATASET types for imaging techniques in breast cancer $[4,5,8]$

\begin{tabular}{|c|c|c|c|}
\hline Imaging type & Advantages & Disadvantages & Features \\
\hline $\begin{array}{l}\text { Mammography } \\
\text { MIAS, DDSM, } \\
\text { INBREAST, } \\
\text { BCDR }\end{array}$ & $\begin{array}{l}\text { Detects micro } \\
\text { calcifications, } \\
\text { uses low x- } \\
\text { Ray doses }\end{array}$ & $\begin{array}{l}\text { False alarm, low } \\
\text { contrast, less in } \\
\text { case of dense } \\
\text { breasts }\end{array}$ & $\begin{array}{l}\text { Standardized } \\
\text { method for } \\
\text { detection of } \\
\text { early onset of } \\
\text { breast cancer }\end{array}$ \\
\hline $\begin{array}{c}\text { MRI } \\
\text { Breast-MRI- } \\
\text { NACT-Pilot } \\
\text { QIN-Breast } \\
\text { Mouse- } \\
\text { Mammary } \\
\end{array}$ & $\begin{array}{l}\text { Non-invasive, } \\
\text { high temporal } \\
\text { resolution }\end{array}$ & $\begin{array}{c}\text { Memory } \\
\text { requirement, } \\
\text { patient } \\
\text { discomfort }\end{array}$ & $\begin{array}{l}\text { Detects breast } \\
\text { cancer }\end{array}$ \\
\hline Thermography & Non-invasive & $\begin{array}{c}\text { Low resolution } \\
\text { of infrared } \\
\text { cameras }\end{array}$ & $\begin{array}{l}\text { Suitable for } \\
\text { muscle tissue }\end{array}$ \\
\hline Ultrasound & $\begin{array}{l}\text { Quick, Highly } \\
\text { sensitive, } \\
\text { widely } \\
\text { accessible }\end{array}$ & $\begin{array}{c}\text { Technician } \\
\text { sensitive, } \\
\text { quality depends } \\
\text { on personnel } \\
\text { doing the scan } \\
\end{array}$ & $\begin{array}{l}\text { For dense and } \\
\text { soft tissues }\end{array}$ \\
\hline $\begin{array}{c}\text { Histopathology } \\
\text { TCGA-BRCA } \\
\text { BreakHis }\end{array}$ & $\begin{array}{l}\text { High spectral } \\
\text { features, easy } \\
\text { segmentation }\end{array}$ & $\begin{array}{c}\text { Does not } \\
\text { provide a } \\
\text { complete } \\
\text { diagnostic } \\
\text { picture } \\
\end{array}$ & $\begin{array}{l}\text { Extensively } \\
\text { worked upon }\end{array}$ \\
\hline
\end{tabular}

Amongst the various established machine learning based methods most popular methods include support vector machines, convolutional neural networks, genetic algorithm as optimizer, naïve Bayes classifier, Artificial neural networks, nearest neighbours, random forest and decision trees. The major underlying criterion for an efficient method is to aim at retrieval of intrinsic features of suspicious lesions. Based on the performance and type of feature selection methods we aim at outlining a brief review of various ML-CAD tools for breast cancer detection.

\section{RECENT TRENDS IN BREAST CANCER DETECTION}

The procedure for employing a pre-trained machine learning CAD model in order to determine the malignancy of a lesion entails: (i) outlining of a suspicious region or region of interest (ROI); (ii) Feature extraction is used to quantify the ROI with scalar variables; (iii) feature selection is necessary to lower the feature dimension and to facilitate the retrieval of information; (iv) output of the labelled lesion is predicted using ML as classifier output $[4,5]$.
In a recent work in 2019 documented in [10], a convolutional network based method for the classification of the screening mammograms was developed using full field digital mammography using INbreast database and Digital Database. This method was able to detect the prevalence of cancer in breast on mammogram screening. The "end to end" approach employed in this method was capable of leveraging the training data sets with only the label of cancer or with full clinical annotation. This method by achieving high level of accuracy on heterogeneous mammograms holds to deliver improved clinical tools with reduced false mammography results. In another deep CNN based approach, examination of histopathological images has been presented using Inception_V3 and Inception_ResNet_V2 trained with transfer learning techniques. The model is trained to perform binary as well multi-class classification of images with breast cancer by re-training the parameters in the fully connected layer. The experimental methods depict that this trained architecture is able to extract higher informative level of features and Inception_ResNet_V2 network based deep transfer learning is a potential method for analysis of histopathological images [11].

Amongst the artificial intelligence based methods, SVM (Support Vector Machine) is most commonly employed technique for breast cancer detection. This technique inspired by statistical learning theory has incorporated the machine learning set. Besides able to operate on optional features eliminating the need to create independent hypotheses, this method is also able to reduce the over-fitting problem in the training data [4]. In case of fuzzy SVM, the fuzzy membership function is employed to weigh each sample in the training set. This method assures that each sample is weighed by one or more classes. In [12] a fuzzy SVM based machine learning method is presented in order to detect breast cancer in ultrasound images. This method combines textural and morphological features and delivers an accuracy of $95.85 \%$ and sensitivity of $91.46 \%$. Another deep learning approach is given in [13] to detect breast lesions and nodules in ultrasound images. As a part of pre-classifier stage this method employs equalization of histogram, image cropping and marginal augmentation. For classifying, this method uses CNN based architecture. The authors claim that that classification of malignant sets showed promising results.

With an outstanding effect of outperforming the Tyrer-Cusick model (version 8)-a latest clinical standard, authors in [14] designed three stage deep learning model that employs conventional risk factors (patient questionnaire and medical records to assess breast cancer. This method affirmed the finding that where mammography data entails information indicating risk, DL method is able to efficiently deduce this data. In order to match the decision accuracy given by an expert panel of radiologists, a multi-view DCNN based method has been presented in [15]. Due to the presence of fine intricate features in medical images, the existing architecture is inadequate and are trained for natural images. Besides, these architectures work on heavily downsized image which limit the performance in case of medical images to a large extent. The.

$$
\alpha+\beta=\chi
$$




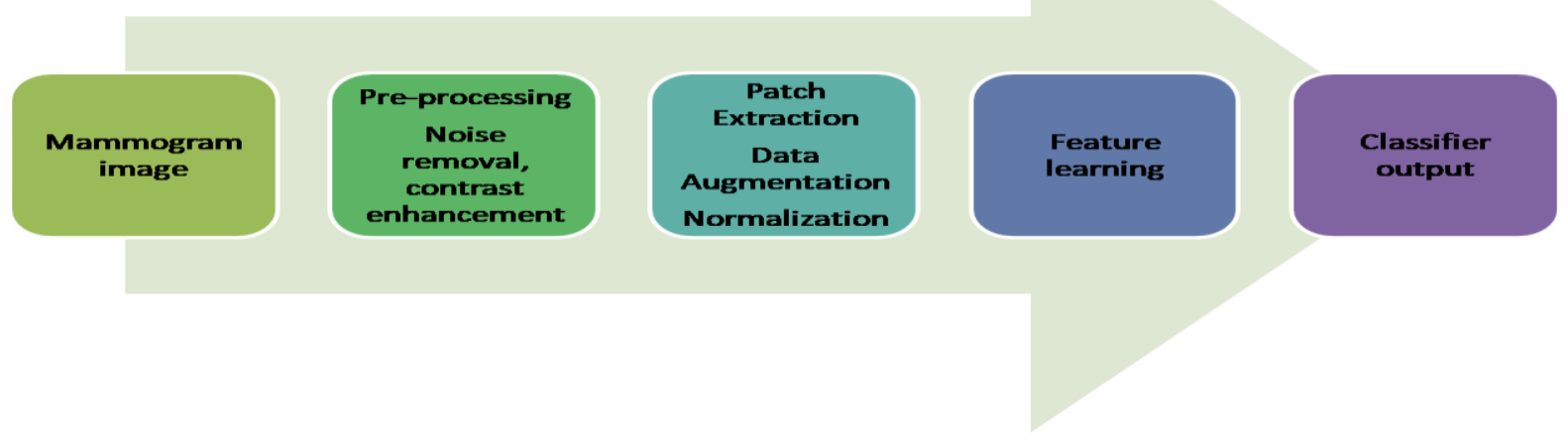

Fig2: Block Diagram of a basic CAD tool

Table 2: Various methods employed in different stages of ML-CAD tools [8]

\begin{tabular}{|c|c|}
\hline \multirow[t]{4}{*}{ Pre-processing } & $\begin{array}{l}\text { Bad Pixel: anomalous pixel depicting abrupt behaviour from the rest of the array; a black or } \\
\text { white pixel; replaced by average of neighbouring pixels }\end{array}$ \\
\hline & $\begin{array}{l}\text { Noise removal: Most important pre-processing technique. e.g gabor filter, morphological } \\
\text { operations, Non-local mean filter }\end{array}$ \\
\hline & $\begin{array}{l}\text { Vignetting: source of noise in thermograms, generates a darkening effect; occurs due to } \\
\text { temperature difference in ambient area of affected region }\end{array}$ \\
\hline & $\begin{array}{l}\text { Temperature calibration: In case of infrared images grey scale values into a temperature } \\
\text { function }\end{array}$ \\
\hline \multirow[t]{4}{*}{ Image segmentation } & $\begin{array}{l}\text { Edge Detection method: detects the discontinuities and sharp edges; not suitable for low } \\
\text { contrast details. }\end{array}$ \\
\hline & $\begin{array}{l}\text { Region dependent method: employed where pixel in nearby are similar, separation and } \\
\text { assembly of target area; large memory requirement }\end{array}$ \\
\hline & $\begin{array}{l}\text { Thersholding method: suitable for images with well defined edges; computationally } \\
\text { efficient; no need of prior knowledge }\end{array}$ \\
\hline & $\begin{array}{l}\text { Fuzzy method: employs fuzzy operators and mathematics; suitable in linguistic phrases, } \\
\text { computationally complex }\end{array}$ \\
\hline & $\begin{array}{l}\text { Neural networks: used for clustering and categorization; requires large amount of time for } \\
\text { training }\end{array}$ \\
\hline Feature extraction & $\begin{array}{l}\text { Mean, Entropy, homogeneity, correlation, coarseness, kurtosis, energy, variance, standard } \\
\text { deviation, contrast, line likeness }\end{array}$ \\
\hline
\end{tabular}

method proposed in this work was evaluated on large scale of 886000 mammographic images. This method reaffirms the idea that usage of full size medical images is imperative to increased performance of network architecture. The level of performance and accuracy of a classifier is largely affected by the quality of the raw input images. The input images are corrupted with noise and contrast adjustment is required in order to avoid misclassifications in the final result. In a work given in [16] mammograms are pre-processed using morphological operations and gabor filtering. A deep learning based breast cancer detection model has been presented using SVM, CNN and Random Forest as classifiers. This method suggests that CNN are a better tool for high level of breast lesion detection. Machine learning algorithms have taken a hold across the oncology community to generate classification based prognostic models to monitor progression of cancer stages and survivability. Particularly semi-supervised learning machine learning methods have been designed for the breast cancer detection on SEER (Surveillance Program, Epidemiology, and End Results) datasets. However these methods do not take into consideration of the proliferation index. In order to harness the attributes of wide range of methods, a combined approach of machine learning and random optimization which seeks decision and interpretability both together has been designed [17]. Another approach given in [18] presents an unsupervised learning that can carry large number of prognostic features into consideration.
A large amount of literature has been documented in context of breast cancer diagnose and detection on varied type of dataset. Most of the methods designed for histological images are deep learning based for instance: UNet and AlexNet and are capable of automatic learning which is representative of data [19-22]. Another well known methods include combinational approaches of Deep learning networks and SVM, Adaboost and Random forest classifiers. Many a times even established researchers/ design teams opt for different training strategies for a specific task using the same data set and similar network architecture and end up obtaining widely different results [23, 24].

A CNN based method ISB2016 (27 layers) gave an outstanding performance for the detection of metastasis. An approach to distinguish between malignant and benign tissues was presented using $\mathrm{CNN}$ based on AlexNet [25]. A multi-classification method was employed to develop three configurations of ResNet in order to classify ICIAR2018 BACH challenge. Besides these CNN trained end to end classification techniques, other popular approaches are unsupervised networks which are auto-encoder (AE) based. AE are primarily employed for pre-training of neural networks and are thoroughly optimized to learn the principal features of the data. In [26], a sparse AE representation is given in order to identify the regions which are closely related to basal cell carcinoma detection. Other than this a stacked sparse AE framework has been given in [27] for the automatic detection of nucleus in breast cancer dataset. In order to target two cases of applications i.e. lymphoma subtype classification and invasive ductal carcinoma, a residual convolutional neural 
network model derived from FusionNet and auto-encoder based has been presented in [19]. The method has been trained to automatically analyse Hemotoxylin and Eosin (H\&E) stained histopathical images. In [28], a system is proposed which integrates breast segmentation model with region based convolutional network to match the classification accuracy of BI-RADS score. Another CNN based approach is given in [29] on similar dataset

Different segmentation approaches affect the efficacy of a classifying algorithm. In order to classify benign and malignant lesions in breast mammograms a deep learning based algorithm involving two methods for segmentation has been proposed in [30]. The two approaches follows manual determination of region of interest and threshold based segmentation respectively. The method trained using AlexNet is able to achieve considerable level of accuracy as last fully connected layered is joined to SVM classifier. A fully convolutional auto-encoder based approach has been presented in [31] for invasive carcinoma diagnosis in H\&E stained breast images. This method presents a pattern mining approach by learning the contrast pattern differences in malignant and benign tissues. The evaluation of the presented method is carried out on a publicly available data set and was able to detect the malignant cluster cells in an image.

Besides these highly efficient methods, a computationally simple and easy wavelet neural network based approach has been given in [32]. This method presents an improvisation of existing artificial neural network based methods by employing wavelets as the activation function. Amongst various new surfacing ideas for breast cancer detection, another interesting approach presents processing of the routine blood analysis by employing machine learning algorithms such as Artificial Neural Networks, SVM, K-nearest neighbour and standard extreme learning Machine [33]. With an attempt to design an efficient segmentation technique authors in [34] extracted the cancerous region from thermograms by combining the Firefly algorithm assisted Kapur's entropy and Hidden Markov random field. The results obtained are highly promising and give better quality as compared to Distance Regularized Level Set (DRLS).On similar lines a work given in [35] presents a hybrid framework in order to assess the breast abnormality. This method combines together SGO+KE (social group optimization and kapur's entropy) thresholding to extort and examine the breast abnormality and also to group the similar pixel values. With the help of fuzzy $\mathrm{C}$-mean clustering the threshold result is segmented into three regions. The proposed method is able to exhibit an exceptional performance of DITI India and Visual Lab dataset. In the terms of emerging technologies for breast lesion techniques, microwave imaging due to their dielectric properties gives a safe and non-ionizing platform to distinguish between unhealthy and healthy issues. In microwave imaging microwave signals are transmitted through the tissue and dedicated receiving and transmitting antenna set up gathers the scattering parameters. In [36], a successful attempt has been made to augment machine learning with microwave engineering for breast cancer detection. The various machine learning algorithms like SVM and Multi-layered perceptron have been trained which are able to achieve high level of accuracy for lesion the detection. A work given in [49] classifies BreakHis data set using DNN based techniques instructed by statistical and structural information. This method specifically employs CNN, a Long-Short-Term-Memory and their combination for breast cancer detection. The method with the help of softmax and support vector machine is able give a high level of accuracy. In [50] authors developed an integrated approach of machine learning and deep learning model which is trained on health records and linked mammograms. The method obtained an acceptable performance as compared to that of the radiologists. Under the hierarchy of artificial neural networks feed forward back propagation model (FFBPN) has the typical characteristic that each layer of neuron is linked to the previous layer of neurons. A work given in [51] employs a FFBPN for categorization of breast cancer images into benign and malignant. The method was evaluated using Wisconsin Breast cancer database. The network designed with three hidden layers; 21 neurons in hidden layer perform better as compared to other existing methods. Besides these various other deep learning methods can be found in [52].

\begin{tabular}{|c|c|c|}
\hline Method & Ref. No & Pros and Cons \\
\hline CNN & {$[10]$} & Inbreast dataset; high accuracy; provide leverage to the training data; huge memory \\
\hline K-means clustering & [11] & Extract intricate features, noise issues result in significant difference in contrast resolution \\
\hline Deep learning & [14] & Increased level of accuracy; requires training on extended level \\
\hline CNN & [17] & Matching expert level diagnosis, FFDM, large memory requirement \\
\hline SVM & {$[4,37]$} & Information measure of correlation, use of curvelet prior to feature extraction; thermal images, \\
\hline 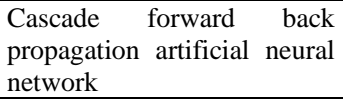 & {$[4,38]$} & Mammography; employs auto-correlation; probability; Entropy; Feature extraction from known database \\
\hline A 3 layered CNN & {$[5,39]$} & Analysis of image in transform domain and SVM based classification \\
\hline $\begin{array}{l}\text { Transfer learning method } \\
\text { AlexNet }\end{array}$ & {$[5,40]$} & Compared with other CNN models; Data augmentation \\
\hline A 7 layered CNN model & {$[5,41]$} & Parameters are initialized; Parasitic metric learning \\
\hline $\begin{array}{c}\text { Transferred VGG (deep } \\
\text { neural network) }\end{array}$ & [42] & performed better than GoogleNet \\
\hline $\begin{array}{c}\text { Transferred } \\
\text { VGG/ResNet/Inception } \\
\end{array}$ & [43] & systematic comparison, time consuming \\
\hline $\mathrm{K}-\mathrm{NN}$ & [44] & Designs a input output mapping function; uses extraction feature like gray level non uniformity \\
\hline Deep learning & [45] & $\begin{array}{l}\text { Mammogram based method; classifies malignant and benign masses; minimal error; direct application; do not } \\
\text { require human intervention; depends heavily on ROI curve. }\end{array}$ \\
\hline $\begin{array}{l}\text { Feed-Forward propagation } \\
\text { artificial neural network }\end{array}$ & [46] & Mammography; employs image registration methods \\
\hline Naïve Bayes & [47] & Requires small amount of data to estimate the necessary parameters; less accurate than SVM \\
\hline Genetic algorithm & [48] & Less accurate \\
\hline
\end{tabular}




\section{DISCUSSION AND CONCLUSION}

Witnessing a high mortality rate due to breast cancer the need for automatic computer aided diagnostic tools is quite clear. These CAD tools are being regularly employed by radiologists and its impact on the research community is quite evident with large amount of literature. This method acts as a second interpreter and eventually strengthens the radio-diagnosis by reducing the false positives. The reduction in number of false positives is more important as it results in unnecessary discomfort, cost and hazards of biopsy. CAD tools progressively abridge the time between patient care and management by reviewing and reporting the outcomes in few seconds. A large number of novel and intuitive methods have been given in literature, but none of the method is able suffice for all cancer and lesion scenarios. This article has laid down a general framework employed in a classifier approach for breast cancer detection along with the recent trends in the field. According to the brief survey it can be stated that Support vector machine based classifiers are able to perform with extended level of accuracy and precision. SVM is a popular and established method based on statistical learning theory. The following properties of SVM make them extremely popular in CAD analysis (I) optimal subset for learning; employs v-fold cross validation approach; high number of spilt pages ensures higher differentiation. However this method also has some limitations as it requires large number of training data set; requirement of choosing appropriate kernels and need to select one or more parametric models to train the network. Besides this the convolutional neural networks are also giving state-of-the-art performance in terms of accuracy and precision. It is being found that the quality of the input images largely affects the performance of the complete model. For instance the pre-processing of source images for noise removal and contrast enhancement plays a major role in increasing the efficacy of the trained model as noise prevalence can lead to increased chances of false diagnosis. However in terms employment of noise removal methods in CAD tools, not much attention has been given. It seems in hoard of designing highly efficient classifiers, the researchers tend to miss on to the implementation of efficient denoising methods available in literature such as BM3D, Nonlocal Mean Filter and Patch based self similarity methods for pre-processing. Although this may lead to a slight increase in computational burden but the accuracy may increase manifold. Similar is the case with the segmentation methods. These employed methods hold a lot of margin for improvement. The optimization based segmentation methods find the emerging platform for effective segmentation of region of interests. Also the methods feature extract could be more robust in terms of feature selection. Including large number of features induces redundancy which can be addressed by employing fusion of significant feature details as selected features. Besides this Full-field digital mammography methods holds a lot of potential for future of breast cancer detection as they are able to provide high resolution images. However these methods require a large number of training data set and hence large memory requirement. Therefore focus on pre-processing methods, high resolution data and CNN can further amplify our progress in the field of breast cancer detection.

\section{REFERENCES}

[1] CONSENSUS DOCUMENT FOR MANAGEMENT OF BREAST CANCER, Indian Council of Medical Research, Ansari Nagar, New Delhi 2016

[2] Løberg, Magnus, Mette Lise Lousdal, Michael Bretthauer, and Mette Kalager. "Benefits and harms of mammography screening." Breast Cancer Research 17, no. 1 (2015): 63.

[3] Nahid, Abdullah-Al, and Yinan Kong. "Involvement of machine learning for breast cancer image classification: a survey." Computational and mathematical methods in medicine 2017 (2017).

[4] Sadoughi, Farahnaz, Zahra Kazemy, Farahnaz Hamedan, Leila Owji, Meysam Rahmanikatigari, and Tahere Talebi Azadboni. "Artificial intelligence methods for the diagnosis of breast cancer by image processing: a review." Breast Cancer: Targets and Therapy 10 (2018): 219.

[5] Zou, Lian, Shaode Yu, Tiebao Meng, Zhicheng Zhang, Xiaokun Liang, and Yaoqin Xie. "A Technical Review of Convolutional Neural Network-Based Mammographic Breast Cancer Diagnosis." Computational and mathematical methods in medicine 2019 (2019).

[6] Nover, Adam B., Shami Jagtap, Waqas Anjum, Hakki Yegingil, Wan Y. Shih, Wei-Heng Shih, and Ari D. Brooks. "Modern breas cancer detection: a technological review." Journal of Biomedical Imaging 2009 (2009): 26.

[7] Warren Burhenne, Linda J., Susan A. Wood, Carl J. D'Orsi, Stephen A. Feig, Daniel B. Kopans, Kathryn F. O'Shaughnessy, Edward A. Sickles, Laszlo Tabar, Carl J. Vyborny, and Ronald A Castellino. "Potential contribution of computer-aided detection to the sensitivity of screening mammography." Radiology 215 , no. 2 (2000): 554-562.

[8] Munir, Khushboo, Hassan Elahi, Afsheen Ayub, Fabrizio Frezza, and Antonello Rizzi. "Cancer Diagnosis Using Deep Learning: A Bibliographic Review." Cancers 11, no. 9 (2019): 1235

[9] https://breast-cancer.ca/mrifacts/ accessed om 12.07.2019

[10] Shen, Li, Laurie R. Margolies, Joseph H. Rothstein, Eugene Fluder, Russell McBride, and Weiva Sieh. "Deep Learning to Improve Breast Cancer Detection on Screening Mammography." Scientific reports 9 (2019).

[11] Xie, Juanying, Ran Liu, I. V. Luttrell, and Chaoyang Zhang. "Deep Learning Based Analysis of Histopathological Images of Breast Cancer." Frontiers in genetics 10 (2019): 80

[12] Karahaliou, A., Skiadopoulos, S., Boniatis, I., Sakellaropoulos, P., Likaki, E., Panayiotakis, G. and Costaridou, L., 2007. Texture analysis of tissue surrounding microcalcifications on mammograms for breast cancer diagnosis. The British journal of radiology, 80(956), pp.648-656

[13] Han, Seokmin, Ho-Kyung Kang, Ja-Yeon Jeong, Moon-Ho Park, Wonsik Kim, Won-Chul Bang, and Yeong-Kyeong Seong. "A deep learning framework for supporting the classification of breast lesions in ultrasound images." Physics in Medicine \& Biology 62, no. 19 (2017): 7714.

[14] Yala, Adam, Constance Lehman, Tal Schuster, Tally Portnoi, and Regina Barzilay. "A deep learning mammography-based model for improved breast cancer risk prediction." Radiology (2019): 182716.

[15] Geras, Krzysztof J., Stacey Wolfson, Yiqiu Shen, Nan Wu, S. Kim, Eric Kim, Laura Heacock, Ujas Parikh, Linda Moy, and Kyunghyun Cho. "High-resolution breast cancer screening with multi-view deep convolutional neural networks." arXiv preprint arXiv:1703.07047 (2017).

[16] "Vasundhra et al., "Machine Learning Approach for Breast cancer detection", International journal of recent technology and engineering", May 2019.

[17] Ferroni, Patrizia, Fabio M. Zanzotto, Silvia Riondino, Noemi Scarpato, Fiorella Guadagni, and Mario Roselli. "Breast Cancer Prognosis Using a Machine Learning Approach." Cancers 11, no. 3 (2019): 328.

[18] Hueman, Mathew T., Huan Wang, Charles Q. Yang, Li Sheng, Donald E. Henson, Arnold M. Schwartz, and Dechang Chen. "Creating prognostic systems for cancer patients: A demonstration using breast cancer." Cancer medicine 7 , no. 8 (2018): 3611-3621. 
[19] Brancati, Nadia, Giuseppe De Pietro, Maria Frucci, and Daniel Riccio. "A Deep Learning Approach for Breast Invasive Ductal Carcinoma Detection and Lymphoma Multi-Classification in Histological Images." IEEE Access 7 (2019): 44709-44720

[20] He, Kaiming, Xiangyu Zhang, Shaoqing Ren, and Jian Sun. "Deep residual learning for image recognition." In Proceedings of the IEEE conference on computer vision and pattern recognition, pp. 770-778. 2016.

[21] Krizhevsky, Alex, Ilya Sutskever, and Geoffrey E. Hinton. "Imagenet classification with deep convolutional neural networks." In Advances in neural information processing systems, pp. 1097-1105. 2012

[22] Ronneberger, O., P. Fischer, and TU-net Brox. "Convolutional networks for biomedical image segmentation." In Paper presented at: International Conference on Medical Image Computing and Computer-Assisted Intervention2015.

[23] Aresta, Guilherme, Teresa Araújo, Scotty Kwok, Sai Saketh Chennamsetty, Mohammed Safwan, Varghese Alex, Bahram Marami et al. "Bach: Grand challenge on breast cancer histology images." Medical image analysis (2019).

[24] Liu, Yun, Krishna Gadepalli, Mohammad Norouzi, George E. Dahl, Timo Kohlberger, Aleksey Boyko, Subhashini Venugopalan et al. "Detecting cancer metastases on gigapixel pathology images." arXiv preprint arXiv:1703.02442 (2017).

[25] Vu, Tiep Huu, Hojjat Seyed Mousavi, Vishal Monga, Ganesh Rao, and UK Arvind Rao. "Histopathological image classification using discriminative feature-oriented dictionary learning." IEEE transactions on medical imaging 35, no. 3 (2015): 738-751.

[26] Cruz-Roa, Angel Alfonso, John Edison Arevalo Ovalle, Anant Madabhushi, and Fabio Augusto González Osorio. "A deep learning architecture for image representation, visual interpretability and automated basal-cell carcinoma cancer detection." In International Conference on Medical Image Computing and Computer-Assisted Intervention, pp. 403-410. Springer, Berlin, Heidelberg, 2013.

[27] Xu, Jun, Lei Xiang, Qingshan Liu, Hannah Gilmore, Jianzhong Wu, Jinghai Tang, and Anant Madabhushi. "Stacked sparse autoencoder (SSAE) for nuclei detection on breast cancer histopathology images." IEEE transactions on medical imaging 35, no. 1 (2015): 119-130.

[28] Akselrod-Ballin, Ayelet, Leonid Karlinsky, Sharon Alpert, Sharbell Hashoul, Rami Ben-Ari, and Ella Barkan. "A CNN based method for automatic mass detection and classification in mammograms." Computer Methods in Biomechanics and Biomedical Engineering: Imaging \& Visualization 7, no. 3 (2019): 242-249.

[29] Araújo, Teresa, Guilherme Aresta, Eduardo Castro, José Rouco, Paulo Aguiar, Catarina Eloy, António Polónia, and Aurélio Campilho. "Classification of breast cancer histology images using convolutional neural networks." PloS one 12, no. 6 (2017): e0177544.

[30] Ragab, Dina A., Maha Sharkas, Stephen Marshall, and Jinchang Ren. "Breast cancer detection using deep convolutional neural networks and support vector machines." PeerJ 7 (2019): e6201.

[31] Li, Xingyu, Marko Radulovic, Ksenija Kanjer, and Konstantinos N. Plataniotis. "Discriminative Pattern Mining for Breast Cancer Histopathology Image Classification via Fully Convolutional Autoencoder." IEEE Access 7 (2019): 36433-36445.

[32] Dheeba, V., N. Albert Singh, and J. Amar Pratap Singh. "Breast Cancer Diagnosis: An Intelligent Detection System Using Wavelet Neural Network." In Proceedings of the International Conference on Frontiers of Intelligent Computing: Theory and Applications (FICTA) 2013, pp. 111-118. Springer, Cham, 2014.

[33] Aslan, Muhammet Fatih, Yunus Celik, Kadir Sabanci, and Akif Durdu. "Breast cancer diagnosis by different machine learning methods using blood analysis data." International Journal of Intelligent Systems and Applications in Engineering 6, no. 4 (2018): 289-293.

[34] Raja, N., V. Rajinikanth, Steven Lawrence Fernandes, and Suresh Chandra Satapathy. "Segmentation of breast thermal images using Kapur's entropy and hidden Markov random field." Journal of Medical Imaging and Health Informatics 7, no. 8 (2017): 18251829

[35] Fernandes, Steven Lawrence, Venkatesan Rajinikanth, and Seifedine Kadry. "A Hybrid Framework to Evaluate Breast
Abnormality Using Infrared Thermal Images." IEEE Consume Electronics Magazine 8, no. 5 (2019): 31-36.

[36] Rana, Soumya Prakash, Maitreyee Dey, Gianluigi Tiberi, Lorenzo Sani, Alessandro Vispa, Giovanni Raspa, Michele Duranti, Mohammad Ghavami, and Sandra Dudley. "Machine Learning Approaches for Automated Lesion Detection in Microwave Breast Imaging Clinical Data." Scientific reports 9, no. 1 (2019): 10510.

[37] Francis, Sheeja V., M. Sasikala, and S. Saranya. "Detection of breast abnormality from thermograms using curvelet transform based feature extraction." Journal of medical systems 38, no. 4 (2014): 23

[38] Saini, Satish, and Ritu Vijay. "Mammogram analysis using feedforward back propagation and cascade-forward back propagation artificial neural network." In 2015 Fifth International Conference on Communication Systems and Network Technologies, pp. 1177 1180. IEEE, 2015

[39] Jadoon, M. Mohsin, Qianni Zhang, Ihsan Ul Haq, Sharjeel Butt, and Adeel Jadoon. "Three-class mammogram classification based on descriptive $\quad \mathrm{CNN}$ features." BioMed research international 2017 (2017).

[40] Zhang, Xiaofei, Yi Zhang, Erik Y. Han, Nathan Jacobs, Qiong Han, Xiaoqin Wang, and Jinze Liu. "Classification of whole mammogram and tomosynthesis images using deep convolutional neural networks." IEEE transactions on nanobioscience 17, no. 3 (2018): 237-242.

[41] Jiao, Zhicheng, Xinbo Gao, Ying Wang, and Jie Li. "A parasitic metric learning net for breast mass classification based on mammography." Pattern Recognition 75 (2018): 292-301

[42] Perre, Ana C., Luís A. Alexandre, and Luís C. Freire. "Lesion classification in mammograms using convolutional neural networks and transfer learning." Computer Methods in Biomechanics and Biomedical Engineering: Imaging \& Visualization (2018): 1-7.

[43] Chougrad, Hiba, Hamid Zouaki, and Omar Alheyane. "Deep convolutional neural networks for breast cancer screening." Computer methods and programs in biomedicine 157 (2018): 19-30

[44] Acharya, U. Rajendra, Eddie Yin-Kwee Ng, Jen-Hong Tan, and S. Vinitha Sree. "Thermography based breast cancer detection using texture features and support vector machine." Journal of medical systems 36, no. 3 (2012): 1503-1510.

[45] Qiu, Yuchen, Shiju Yan, Rohith Reddy Gundreddy, Yunzhi Wang, Samuel Cheng, Hong Liu, and Bin Zheng. "A new approach to develop computer-aided diagnosis scheme of breast mass classification using deep learning technology." Journal of $X$ ray Science and Technology 25, no. 5 (2017): 751-763.

[46] Saini, Satish, and Ritu Vijay. "Optimization of Artificial Neural Network Breast Cancer Detection System based on Image Registration Techniques." International Journal of Computer Applications 105, no. 14 (2014): 26-29.

[47] Milosevic, Marina, Dragan Jankovic, and Aleksandar Peulic. "Thermography based breast cancer detection using texture features and minimum variance quantization." EXCLI journal 13 (2014): 1204.

[48] Haddadnia, Javad, Maryam Hashemian, and Kazem Hassanpour. "Diagnosis of breast cancer using a combination of genetic algorithm and artificial neural network in medical infrared thermal imaging." Iranian Journal of Medical Physics 9, no. 4 (2012): 265-274.

[49] Nahid, Abdullah-Al, Mohamad Ali Mehrabi, and Yinan Kong. "Histopathological breast Cancer image classification by deep neural network techniques guided by local clustering." BioMed research international 2018 (2018).

[50] Akselrod-Ballin, Ayelet, Michal Chorev, Yoel Shoshan, Adam Spiro, Alon Hazan, Roie Melamed, Ella Barkan et al. "Predicting Breast Cancer by Applying Deep Learning to Linked Health Records and Mammograms." Radiology (2019): 182622.

[51] Abdel-Ilah, Layla, and Hana Šahinbegović. "Using machine learning tool in classification of breast cancer." In $C M B E B I H$ 2017, pp. 3-8. Springer, Singapore, 2017.

[52] Yu, Cuiru, Houjin Chen, Yanfeng Li, Yahui Peng, Jupeng Li, and Fan Yang. "Breast cancer classification in pathological images based on hybrid features." Multimedia Tools and Applications (2019): 1-21. 